\title{
Remarks on Recognizability of Four-Dimensional Topological Components
}

\author{
Makoto Nagatomo, Makoto Sakamoto*, Tatsuma Kurogi, Satoshi Ikeda, \\ Masahiro Yokomichi and Hiroshi Furutani \\ Faculty of Engineering, University of Miyazaki, Miyazaki 889-2192, Japan \\ Takao Ito \\ Institute of Engineering, Hiroshima University, Higashi-Hiroshima, Hiroshima 739-8527, Japan
}

Yasuo Uchida

Department of Business Administration, Ube National College of Technology,Ube, Yamaguchi 755-8555, Japan

Tsunehiro Yoshinaga

Department of Computer Science and Electronic Engineering, Tokuyama college of Technology, Shunan, Yamaguchi 745-8585, Japan

*Corresponding author, E-mail: sakamoto@cs.miyazaki-u.ac.jp

Tel: +81-985-58-7392, Fax: +81-985-58-7392

\begin{abstract}
The study of four-dimensional automata as the computational model of four-dimensional pattern processing has been meaningful. However, it is conjectured that the three-dimensional pattern processing has its our difficulties not arising in two- or three-dimensional case. One of these difficulties occurs in recognizing topological properties of four-dimensional patterns because the four-dimensional neighborhood is more complicated than two- or three-dimensional case. Generally speaking, a property or relationship is topological only if it is preserved when an arbitrary ' rubber-sheet ' distortion is applied to the pictures . For example, adjacency and connectedness are topological ; area, elongatedness, convexity, straightness, etc. are not. In recent years, there have been many interesting papers on digital topological properties. For example, an interlocking component was defined as a new topological property in multi-dimensional digital pictures, and it was proved that no one marker automaton can recognize interlocking components in a three-dimensional digital picture. In this paper, we deal with recognizability of topological components by four-dimensional Turing machines, and investigate some properties.
\end{abstract}

Keywords: digital geometry, interlocking component, one marker automaton, three-dimensional automaton, topological component, Turing machine.

\section{Introduction}

Digital geometry has played an important role in computer image analysis and recognition[3]. In particular, there is a well-developed theory of topological properties such as connectedness and holes for two-dimensional arrays[4]. On the other hand, three-dimensional information processing has also become of increasing interest with the rapid growth of computed tomography, robotics, and so on. Thus it has become desirable to study the geometrical properties such as interlocking components and cavities for three-dimensional arrays[2,5]. In[2], interlocking components was proposed as a new topological property of three-dimensional digital pictures : Let S1 and S2 be two subsets of the same three-dimensional digital picture. $S_{1}$ and $S_{2}$ are said to be interlocked when they satisfy the following conditions: 
(1) $S_{1}$ and $S_{2}$ are toruses,

(2) $S_{1}$ goes through a hole of $S_{2}$,

(3) $S_{2}$ goes through a hole of $S_{1}$. By the way, the question of whether processing four-dimensional digital pattern in much difficult than three-dimensional ones is of great interest from the theoretical and practical standpoints both. In recent years, due to the advances in many application areas much as computer animation, motion image processing, and so both, the study of four-dimensional pattern processing has been of crucial importance. Thus, it is very interesting to deal with the geometrical propertises such as interlocking components and cavities in a four-dimensional digital picture.

The interlocking of $S_{1}$ and $S_{2}$ in a four-dimensional tape is illustrated in Fig.1. This relation may be considered as a chainlike connectivity.

It is proved that no one marker automaton can recognize interlocking components in a three-dimensional digital picture in [2]. In this paper, we investigate recognizability of topological properties such as interlocking components by three-dimensional Turing machines.

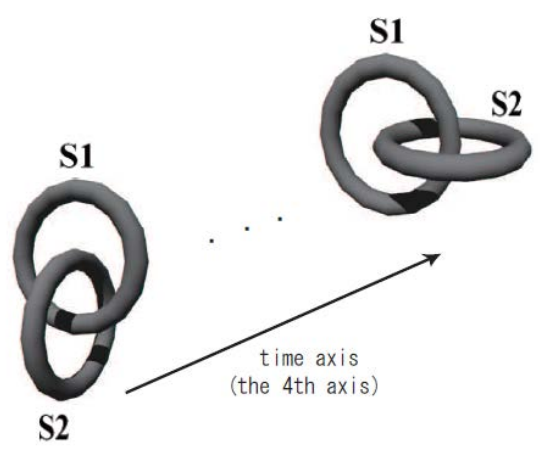

Fig. 1: Interlocking components in a four-dimensional tape.

\section{Preliminaries}

Definition 2.1. Let $\Sigma$ be a finite set of symbols. A four-dimensional tape over $\Sigma$ is a four-dimensional array of elements of $\Sigma$. The set of all four-dimensional tapes over $\Sigma$ is denoted by $\Sigma^{(4)}$. Given a tape $x \in \Sigma^{(4)}$, for each $j(1 \leq j \leq 4)$, we let $l_{j}(x)$ be the length of $x$ along the $j$ th axis. When $1 \leq i_{j} \leq l_{j}(x)$ for each $j(1 \leq j \leq 4)$, let $x\left(i_{1}, i_{2}, i_{3}, i_{4}\right)$ denote the symbol in $x$ with coordinates $\left(i_{1}, i_{2}, i_{3}, i_{4}\right)$, as shown in Fig. 2. Furthermore, we define

$$
x\left[\left(i_{1}, i_{2}, i_{3}, i_{4}\right),\left(i_{1}{ }^{\prime}, i_{2}, i_{3}, i_{4}{ }^{\prime}\right)\right],
$$

when $1 \leq i_{j} \leq i_{j}{ }^{\prime} \leq l_{j}(x)$ for each integer $j(1 \leq j \leq 4)$, as the four-dimensional tape $y$ satisfying the following

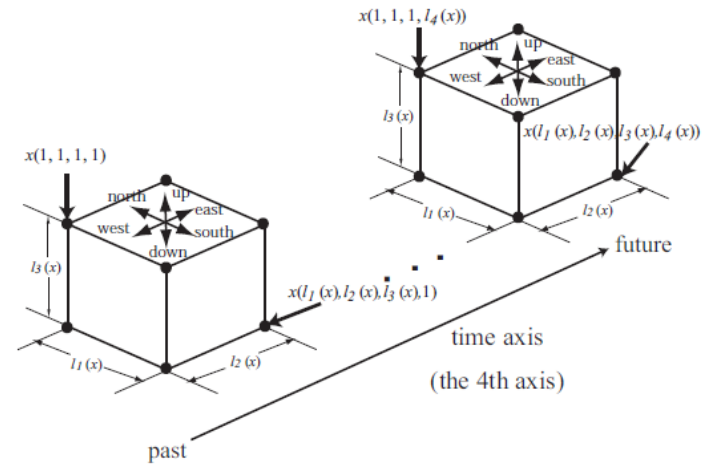

Fig.2 : Four-dimensional input tape.

(i)for each $j(1 \leq j \leq 4), \operatorname{lj}(y)=i_{j}{ }^{\prime}-i_{j}+1$;

(ii) for each $r_{1}, r_{2}, r_{3}, r_{4}\left(1 \leq r_{1} \leq l_{1}(y), 1 \leq r_{2} \leq l_{2}(y), 1 \leq r_{3} \leq l_{3}(y)\right.$, $1 \leq r_{4} \leq l_{4}(y), y\left(r_{1}, r_{2}, r_{3}, r_{4}\right)=x\left(r_{1}+i_{1}-1, r_{2}+i_{2}-1, r_{3}+i_{3}-1\right.$, $\left.r_{4}+i_{4}-1\right)$.

Definition 2.2. A four-dimensional nondeterministic one-marker automaton $4-N M_{1}$ is defined by the six-tuple

$$
M=\left(Q, q_{0}, F, \Sigma,\{+,-\}, \delta\right),
$$

where

(1) $Q$ is a finite set of states;

(2) $q_{0} \in Q$ is the initial state;

(3) $F \subseteq Q$ is the set of accepting states;

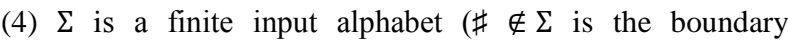
symbol);

(5) $\{+,-\}$ is the pair of signs of presence and absence of the marker; and

(6) $\delta:(Q \times\{+,-\}) \times((\Sigma \cup\{\#\}) \times\{+,-\}) \rightarrow$ $2^{(Q \times\{+,-\})} \times((\Sigma \cup\{\#\}) \times\{+,-\}) \times\{$ east, west, south,

north,up,down,future,past,nomove $\}$ ) is the nextmove function, satisfying the following: For any $q, q^{\prime} \in Q$, any $a, a^{\prime} \in \Sigma$, any $u, u^{\prime}, v, v^{\prime} \in\{+,-\}, \quad$ and any $d \in$ \{east,west,south,north,up,down,future,past,no move\}, if $\left(\left(q^{\prime}, u^{\prime}\right),\left(a^{\prime}, v^{\prime}\right), d\right) \quad \in \quad \delta \quad((q, u),(a, v)) \quad$ then $\quad a=a^{\prime}, \quad$ and $\left(u, v, u^{\prime}, v^{\prime}\right) \in\{(+,-,+,-),(+,-,-,+),(-,+,-,+),(-,+,+,-),(-,-,-,-)\}$.

We call a pair $(q, u)$ in $Q \times\{+,-\}$ an extended state, representing the situation that $M$ holds or does not hold the marker in the finite control according to the sign $u=+$ or $u=$ -, respectively. A pair $(a, v)$ in $\Sigma \times\{+,-\}$ represents an input tape cell on which the marker exists or does not exist according to the sign $v=+$ or $v=-$, respectively. Therefore, 
the restrictions on $\delta$ above imply the following conditions. (A) When holding the marker, $M$ can put it down or keep on holding. (B) When not holding the marker, and (i) if the marker exists on the current cell, $M$ can pick it up or leave it there, or (ii) if the marker does not exist on the current cell, $M$ cannot create a new marker any more.

Definition 2.3. Let $\Sigma$ be the input alphabet of $4-N M_{1} M$. An extended input tape $\tilde{x}$ of $M$ is any four-dimensional tape over $\Sigma \times\{+,-\}$ such that

(i)for each $j(1 \leq j \leq 4), l_{j}(\tilde{x})=l_{j}(x)$,

(ii) for each $i_{1}\left(1 \leq i_{1} \leq l_{1}(\tilde{x})\right), i_{2}\left(1 \leq i_{2} \leq l_{2}(\tilde{x})\right), i_{3}\left(1 \leq i_{3} \leq l_{3}(\tilde{x})\right)$, and $i_{4}\left(1 \leq i_{4} \leq l_{4}(\tilde{x})\right), \tilde{x}\left(i_{1}, i_{2}, i_{3}, i_{4}\right)=x\left(i_{1}, i_{2}, i_{3}, i_{4}, u\right)$ for some $u \in$ $\{+,-\}$.

Definition 2.4. A configuration of $4-N M_{1} M=\left(Q, q_{0}, F, \Sigma, \delta\right)$ is an element of

$$
((\Sigma \cup\{\#\}) \times\{+,-\})^{(4)} \times(Q \times\{+,-\}) \times N^{4},
$$

where $N$ denotes the set of all nonnegative integers. The first component of a configuration $c=\left(\tilde{x},(q, u),\left(i_{1}, i_{2}, i_{3}, i_{4}\right)\right)$ represents the extended input tape of $M$. The second component $(q, u)$ of c represents the extended state. The third component $\left(i_{1}, i_{2}, i_{3}, i_{4}\right)$ of $c$ represents the input head position. If $q$ is the state associated with configuration $c$, then $c$ is said to be an accepting configuration if $q$ is an accepting state. The initial configuration of $M$ on input $x$ is

$$
I_{M}(x)=\left(x^{-},\left(q_{0},+\right),(1,1,1,1)\right),
$$

where $x^{-}$is the special extended input tape of $M$ such that $x^{-}\left(i_{1}\right.$, $\left.i_{2}, \quad i_{3}, \quad i_{4}\right)=\left(x\left(i_{1}, \quad i_{2}, \quad i_{3}, \quad i_{4}\right),-\right)$ for each $i_{1}, \quad i_{2}, \quad i_{3}, \quad i_{4}$ $\left(1 \leq i_{1} \leq l_{1}(\tilde{x}), 1 \leq i_{2} \leq l_{2}(\tilde{x}), 1 \leq i_{3} \leq l_{3}(\tilde{x}), 1 \leq i_{4} \leq l_{4}(\tilde{x})\right)$. If $M$ moves determinately, we call $M$ a fourdimensional deterministic one-marker automaton 4-DM.

Definition 2.5. A seven-way four-dimensional Turing machine is defined by the six-tuple

$$
M=\left(Q, q_{0}, F, \Sigma, \Gamma, \delta\right),
$$

where

(1) $Q$ is a finite set of states;

(2) $q_{0} \in Q$ is the initial state;

(3) $F \subseteq Q$ is the set of accepting states;

(4) $\Sigma$ is a finite input alphabet ( $\# \notin \Sigma$ is the boundary symbol); (5) $\Gamma$ is a finite storage-tape alphabet ( $B \in \Gamma$ is the blank symbol); and

$(6) \delta \subseteq(Q \times(\Sigma \cup\{\#\}) \times \Gamma) \times(Q \times(\Gamma-\{B\}) \times\{$ east,west, south,north,up,down,future,no move $\times \times$ right,left, no move $\}$ ).
If $M$ moves determinately (nondeterminately), we call $M$ a seven-way four-dimensional deterministic (nondeterministic) Turing machine SV 4-DTM (SV 4-NTM). Let $L: N \rightarrow \boldsymbol{R}$ be a function. A seven-way four-dimensional Turing machine $M$ is said to be $L(m)$ space bounded if for all $m \geq 1$ and for each $x$ with $l_{1}(x)=l_{2}(x)=l_{3}(x)=l_{4}(x)=m$, if $x$ is accepted by $M$, then there is an accepting computation path of $M$ on $x$ in which $M$ uses no more than $L(m)$ cells of the storage tape. We denote an $L(m)$ space-bounded $S V$ 4- DTM (SV 4-NTM) by $S V$ 4-DTM(L(m)) (SV 4- NTM $(L(m)))$.

Definition 2.6. Let $T(M)$ be the set of four-dimensional tapes accepted by a machine $M$, and let $£\left[4-D M_{1}\right]=\{T \mid T(M)$ for some 4-DM $M\} . £\left[4-N M_{1}\right]$, etc. are defined in the same way as $£\left[4-D M_{1}\right]$.

We can easily derive the following theorem by using ordinary technique[6].

Theorem 2.1. For any function $L(m) \geq \log m^{3}, \quad £[S V$ $4-N T M(L(m))] \subseteq U c_{>0} £\left[S V 4-D T M\left(2^{c(L(m))}\right)\right]$.

\section{Simulation of four-dimensional one-marker automata by four-dimensional Turing macines}

In this section, we show the algorithms described in the previous section are optimal in some sense. We can get the following Theorems any using the same technique as in the proof of Lemmas 6.2 and 6.3 in [6].

Theorem 3.1. To simulate 4-DM 's, (1) SV 4-NTM's require $\left(m^{3} \log \left(m^{3}\right)\right)$ space and (2) SV 4- DTM's require $\Omega\left(m^{3}\right.$ $\left.\log \left(m^{3}\right)\right)$ spacespace $(m \geq 1)$.

Theorem 3.2. To simulate 4- $N M_{1}$ 's,

(1) SV 4-NTM's require $\Omega\left(m^{6}\right)$ space, and

(2) SV 4-DTM's require $2^{\Omega\left(m^{6}\right)}$ space $(m \geq 1)$.

\section{Recognizability of interlocking components in four-dimensional images}

In this paper, we show that interlocking components are not recognized by any space-bounded four-dimensional Turing machines. First of all, we consider a four-dimensional input tape $T_{3}$ that is 7 units in thickness. So, for some $m$, $T_{3}=\left\{\left(i_{1}, i_{2}, i_{3}, i_{4}\right) \quad \mid 1 \leq i_{1}, i_{2}, i_{3} \leq m+2,1 \leq i_{4} \leq 7\right\}$. 
Fig.3(a)represents $T_{3}$. Now we define two different $5 \times 5 \times 5$ patterns as shown in Fig.3(b)(c). Then we consider an arbitrary $n$-by- $n$ matrix of those $5 \times 5 \times 5$ patterns (see Fig.3).

Then, we can get the following lemma from Lemma 2.1 in [2].

Lemma 4.1. 4-DM 1 cannot recognize interlocking components of an arbitrary given digital picture.

Next, we can get the following lemma by using a technique similar to that in the proof of Lemma 2.1 in [2].

Lemma 4.2. 4- $N D_{1}$ cannot recognize interlocking components of an arbitrary given digital picture.

From Theorem 3.2 and Lemma 4.2, we can get the following.

Theorem 4.1. Interlocking components are not accepted by any $S V$ 4-DTM $(L(m))(S V$ 4-NTM $(L(m)))$ for any function $L(m)$ such that $\lim _{m \rightarrow \infty}\left[L(m) / 2^{m^{6}}\right]=0 \quad\left(\lim _{m} \rightarrow \infty\right.$ $\left.\left[L(m) / m^{6}\right]=0\right)(m \geq 1)$.

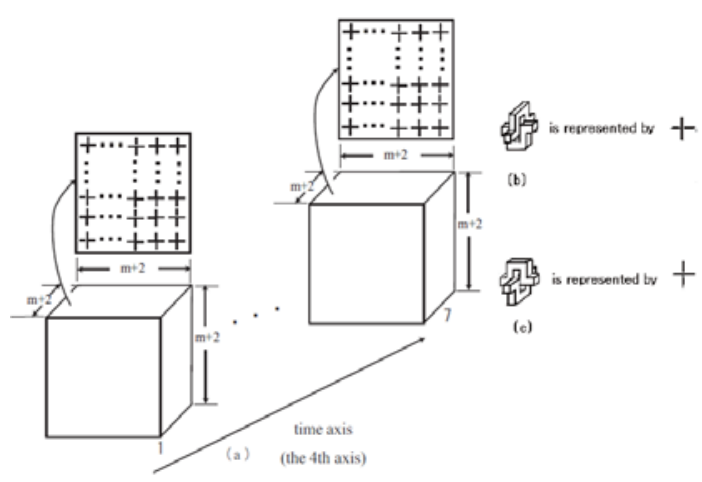

Fig. 3: Four-dimensional input tape including interlocking components $T_{3}[2]$.

\section{Conclusions}

In this paper, we dealt with recognizability of topological components by four-dimensional automata, and showed that interlocking components are not recognized by any space-bounded four-dimensional deterministic or nondeterministic Turing machines. By the way, what is the situation for a two or three marker automata, or for alternation (see [1])? This question seems very interesting. We will investigate the problem in further papers.

\section{References}

1. A.K. Chandra , D.C. Kozen, and L.J. Stockmeyer, Alternation, J. of ACM, 28(1), pp.114-133, 1981.

2. A.Nakamura and K.Aizawa, Detection of ic interlocking components in three-dimensional digital pictures, Imformation Sciences 40, pp.143-153, 1986.

3. A.Rosenfeld and K.C. Kak, Digital Picture Processing, Academic Press, New York, 1976.

4. A.Rosenfeld, Picture Languages, Academic Press, New York, 1979.

5. A.Rosenfeld, Three-dimensionl digital topology, Information and Control 50, pp.119-127, 1981.

6. M.Sakamoto, Three-dimensional alternating Turing machines, Ph. D. Thesis, Y amaguchi University, 1999. 